\title{
On the composite of three irreducible morphisms over string algebras
}

\section{Claudia Chaio}

Depto de Matemática, FCEyN, Universidad Nacional de Mar del Plata, 7600, Mar del Plata, Argentina

E-mail address: claudia.chaio@gmail.com

\section{Flávio U. Coelho}

Departamento de Matemática-IME, Universidade de São Paulo, CP 66281, São Paulo, SP, 05315-970, Brazil

E-mail address: fucoelho@ime.usp.br

\section{Sonia Trepode}

Depto de Matemática, FCEyN, Universidad Nacional de Mar del Plata, 7600, Mar del Plata, Argentina

E-mail address: strepode@mdp.edu.ar

\begin{abstract}
We characterize the representation-finite triangular string algebras having a path of irreducible morphisms of length three between pairwise non-isomorphic modules whose composite lies in the fourth power of the radical.
\end{abstract}

In the study of the module category over an algebra, the so-called irreducible morphisms and their composites play an important role. Recall that a morphism $f: X \longrightarrow Y$, where $X$ and $Y$ are modules over an algebra, is called irreducible provided it does not split and

1991 Mathematics Subject Classification. 16G70, 16G20, 16E10.

Key words: irreducible morphisms, string algebras, infinite radical.

This work is part of the PhD's thesis of the first author and it was supported by CAPES-SECyT, ANPCyT and FAPESP. The second author is a researcher from $\mathrm{CNPq}$ and the third from CONICET. 
whenever $f=g h$, then either $h$ is a split monomorphism or $g$ is a split epimorphism. For indecomposable $A$-modules $X, Y$, denote by $\Re(X, Y)$ the set of all morphisms from $X$ to $Y$ which are not isomorphisms. Observe then that an irreducible morphism from $X$ to $Y$ belongs to $\Re(X, Y)$ but not to its square $\Re^{2}(X, Y)$. However, it is not true, in general, that a composite of $n \geq 2$ irreducible morphisms through indecomposable modules going from $X$ to $Y$ is either zero or lies in $\Re^{n}(X, Y) \backslash \Re^{n+1}(X, Y)$ (see examples in $[6,10]$ ).

In the present paper we continue our study of composite of $n \geq 2$ irreducible morphisms started in $[6,7,8]$. In [6], we considered the situation of when the composite of two irreducible morphisms is nonzero and lies in $\Re^{3}(\bmod A)$ (see also $\left.[1,5]\right)$. In [7], we studied the more general situation of the composite of $n$ irreducible morphisms lying in almost sectional paths, while in [8] we concentrate our attention for the particular case $n=3$.

Continuing our work, here we will characterize the representationfinite triangular string algebras having a path of three irreducible morphisms whose composite is a non-zero morphism in $\Re^{4}$. String algebras were introduced by Butler-Ringel in [4] and the description of its modules given there will be very usufel for the problem we consider here. The analogous case of paths of irreducible morphisms of length two has been considered in [6].

\section{Preliminaries}

1.1. Throughout this paper, all algebras are finite dimensional basic $k$-algebras where $k$ is a fixed algebraically closed field.

A quiver is given by two sets $Q_{0}$ and $Q_{1}$ together with two maps $s, e: Q_{1} \longrightarrow Q_{0}$. The elements of $Q_{0}$ are called points while the elements of $Q_{1}$ are called arrows. For a given arrow $\alpha \in Q_{1}, s(\alpha)$ is the starting point of $\alpha$ and $e(\alpha)$ is the ending point of $\alpha$. For each $\alpha \in Q_{1}$, denote by $\alpha^{-1}$ its formal inverse and define $s\left(\alpha^{-1}\right)=e(\alpha)$ and $e\left(\alpha^{-1}\right)=s(\alpha)$. A walk in $Q$ is $c_{1} \cdots c_{n}$, with $n \geq 1, e\left(c_{i}\right)=s\left(c_{i-1}\right)$ for $1 \leq i \leq n$, and such that $c_{i}$ is either an arrow or the inverse of an arrow. The inverse of a walk $c_{1} \cdots c_{n}$ is $c_{n}^{-1} \cdots c_{1}^{-1}$. Finally, we say that $c_{1} \cdots c_{n}$ is a reduced walk provided $c_{i+1} \neq c_{i}^{-1}$ for each $i$, $1 \leq i \leq n-1$. If $A$ is an algebra, then there exists a quiver $Q_{A}$, called the ordinary quiver of $A$, such that $A$ is the quotient of the path algebra $k Q_{A}$ by an admissible ideal. 
1.2. Let $A$ be an algebra. We denote by $\bmod A$ the category of all finitely generated left $A$-modules, and by ind $A$ the full subcategory of $\bmod A$ consisting of one copy of each isomorphism class of the indecomposable $A$-module. Denote by $\Gamma_{A}$ its Auslander-Reiten quiver, by $\tau$ the Auslander-Reiten translation DTr and by $\tau^{-1}$ its inverse. Let $X \in$ ind $A$. If $X$ is not projective (not injective), denote by $\alpha(X)\left(\alpha^{\prime}(X)\right.$, respectively) the number of indecomposable summands of the middle term of the almost split sequence ending (starting, respectively) at $X$.

A path $X_{1} \longrightarrow X_{2} \longrightarrow \cdots \longrightarrow X_{n-1} \longrightarrow X_{n}$ of irreducible morphisms through indecomposable modules, with $n \geq 3$, is called sectional provided that for each $i=3, \cdots, n, X_{i-2}$ is not isomorphic to $\tau X_{i}$. By [9], the composite of irreducible morphisms lying in a sectional path of length $n$ belongs to $\Re^{n} \backslash \Re^{n+1}$.

1.3. String algebras. A finite dimensional algebra $A=\left(k Q_{A}\right) / I_{A}$ is called string [4] provided:

(S1) Any point of $Q_{A}$ is the starting point of at most two arrows.

(S1') Any point of $Q_{A}$ is the ending point of at most two arrows.

(S2) Given an arrow $\beta$ of $Q_{A}$, there is at most one arrow $\gamma$ with $s(\beta)=e(\gamma)$ and $\beta \gamma \notin I_{A}$.

(S2') Given an arrow $\beta$ of $Q_{A}$, there is at most one arrow $\gamma$ with $e(\beta)=s(\gamma)$ and $\gamma \beta \notin I_{A}$.

(S3) The ideal $I_{A}$ is generated by a set of paths of $Q_{A}$.

1.4. String modules. Let $A=\left(k Q_{A}\right) / I_{A}$ be a string algebra. A string in $Q_{A}$ is either a trivial path $\epsilon_{v}, v \in Q_{0}$, or a reduced walk $C=c_{n} \ldots c_{1}$ of length $n \geq 1$ such that no sub-walk $c_{i+t} \cdots c_{i+1} c_{i}$ nor its inverse belongs to $I_{A}$. We say that a string $C$ starts in a deep (or on a peak) provided there is no arrow $\beta$ such that $C \beta^{-1}(C \beta$, respectively) is a string. Dually, a string $C$ ends in a deep (or on a peak) provided there is no arrow $\beta$ such that $\beta C\left(\beta^{-1} C\right.$, respectively) is a string.

For each string $C=c_{n} \ldots c_{1}$, one defines an indecomposable $A$ module $M(C)$ called string. We refer to [4] for details on this construction, as well for the results mentioned below.

The almost split sequences with indecomposable middle term and involving string modules are described as follows. Given $\beta \in\left(Q_{A}\right)_{1}$ such that there is a string $\gamma_{r}^{-1} \ldots \gamma_{1}^{-1} \beta \delta_{1}^{-1} \ldots \delta_{s}^{-1}$ which starts in a deep 
and ends on a peak (with $r, s \geq 0$ and $\gamma_{i}, \delta_{j}$ being arrows), then such a string is unique. We define the indecomposable string modules $N(\beta)=M\left(\gamma_{r}^{-1} \ldots \gamma_{1}^{-1} \beta \delta_{1}^{-1} \ldots \delta_{s}^{-1}\right), U(\beta)=M\left(\gamma_{r}^{-1} \ldots \gamma_{1}^{-1}\right)$ and $V(\beta)=$ $M\left(\delta_{1}^{-1} \ldots \delta_{s}^{-1}\right)$. Then there exists an almost split sequence $0 \longrightarrow$ $U(\beta) \longrightarrow N(\beta) \longrightarrow V(\beta) \longrightarrow 0$, and each almost split sequence involving string modules with indecomposable middle term is of this form. $11]$.

For unexplained notions on representation theory, we refer to $[2,3$,

\section{Composite of three irreducible morphisms}

2.1. We first recall the following result from [8].

Theorem. Let $A$ be an algebra and let $X_{1}, X_{2}, X_{3}, X_{4} \in$ indA. Then there exist irreducible morphisms $h_{i}: X_{i} \longrightarrow X_{i+1}, i=1,2,3$ such that $h_{3} h_{2} h_{1} \neq 0, h_{3} h_{2} h_{1} \in \Re^{4}\left(X_{1}, X_{4}\right), h_{2} h_{1} \notin \Re^{3}\left(X_{1}, X_{3}\right)$ and $h_{3} h_{2} \notin \Re^{3}\left(X_{2}, X_{4}\right)$ if and only if one of the following conditions is satisfied.

(a) The path $X_{1} \rightarrow X_{2} \rightarrow X_{3}$ is sectional, $\alpha^{\prime}\left(X_{1}\right)=1, \alpha^{\prime}\left(X_{2}\right)=2$ and there are irreducible morphisms $f_{i}: X_{i} \rightarrow X_{i+1}, i=1,2,3$ such that $f_{3} f_{2} f_{1}=0$ and $f_{3} f_{2} \notin \Re^{3}\left(X_{2}, X_{4}\right)$, and a morphism $\varphi \in \Re^{2}\left(X_{3}, X_{3}\right)$ such that $f_{3} \varphi f_{2} f_{1} \neq 0$; or

(b) The path $X_{2} \rightarrow X_{3} \rightarrow X_{4}$ is sectional, $\alpha\left(X_{4}\right)=1, \alpha\left(X_{3}\right)=2$ and there are irreducible morphisms $f_{i}: X_{i} \longrightarrow X_{i+1}, i=$ $1,2,3$ such that $f_{3} f_{2} f_{1}=0$ and $f_{2} f_{1} \notin \Re^{3}\left(X_{1}, X_{3}\right)$, and a morphism $\varphi \in \Re^{2}\left(X_{2}, X_{2}\right)$ such that $f_{3} f_{2} \varphi f_{1} \neq 0$.

2.2. As a consequence, we can easily prove the following.

Proposition. Let $A$ be an algebra and $X_{i} \in$ indA for $i=1, \ldots, 4$, with $X_{2} \nsucceq X_{4}$. Then there exists a path $X_{1} \stackrel{h_{1}}{\longrightarrow} X_{2} \stackrel{h_{2}}{\longrightarrow} X_{3} \stackrel{h_{3}}{\longrightarrow} X_{4}$ of irreducible morphisms such that $h_{3} h_{2} h_{1} \neq 0, h_{3} h_{2} h_{1} \in \Re^{4}\left(X_{1}, X_{4}\right)$, $h_{2} h_{1} \notin \Re^{3}\left(X_{1}, X_{3}\right)$ and $h_{3} h_{2} \notin \Re^{3}\left(X_{2}, X_{4}\right)$ if and only if one of the following conditions is satisfied.

(a) The path $X_{1} \rightarrow X_{2} \rightarrow X_{3}$ is left almost sectional with $\alpha^{\prime}\left(X_{1}\right)=$ $1, \alpha^{\prime}\left(X_{2}\right)=2$ and $\Re\left(X_{1}, X_{4}\right) \neq 0$; or 
(b) The path $X_{2} \rightarrow X_{3} \rightarrow X_{4}$ is right almost sectional with $\alpha\left(X_{4}\right)=1, \alpha\left(X_{3}\right)=2$ and $\Re\left(X_{1}, X_{4}\right) \neq 0$.

Proof. The necessity follows easily from (2.1).

For the sufficiency, assume that (a) holds. Note that $X_{i}$ is not injective for $i=1,2$. Then, by [7] (Theorem 3.5), there exists a path $X_{1} \stackrel{h_{1}}{\longrightarrow}$ $X_{2} \stackrel{h_{2}}{\longrightarrow} X_{3} \stackrel{h_{3}}{\longrightarrow} X_{4}$ of irreducible morphisms such that $0 \neq h_{3} h_{2} h_{1} \in$ $\Re^{4}$. It only remains to prove that $h_{3} h_{2} \notin \Re^{3}$ and $h_{2} h_{1} \notin \Re^{3}$. Clearly, $h_{2} h_{1} \notin \Re^{3}$ because $X_{1} \rightarrow X_{2} \rightarrow X_{3}$ is a sectional path. Now, if $h_{3} h_{2} \in$ $\Re^{3}$, then by [10](Lemma 1.2), $h_{3}$ must be a surjective right minimal almost split morphism, a contradiction to the fact that $\alpha^{\prime}\left(X_{2}\right)=2$. A similar argument for (b) finishes the proof.

Observe that the cases (a) and (b) of the above result induce, respectively, the following configurations in $\Gamma_{A}$ :
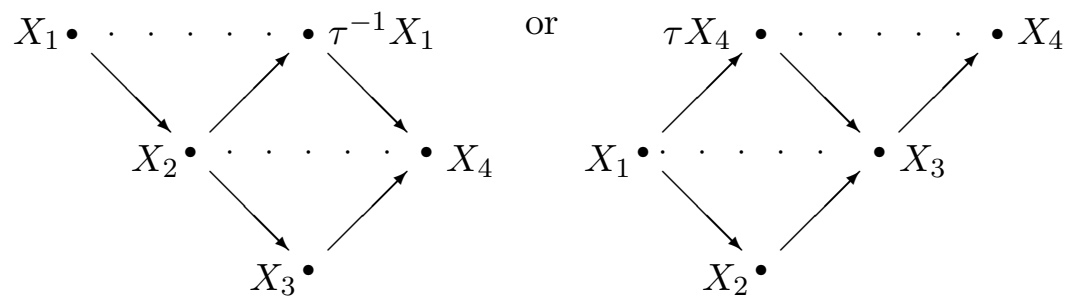

\section{The results}

3.1. In our first result, we give necessary and sufficient conditions for the existence of paths of irreducible morphisms between string modules of length three to have non-zero composite lying in $\Re^{4}$.

Proposition. Let $A=k Q_{A} / I_{A}$ be a connected string algebra and $X_{i} \in$ ind $A, i=1, \cdots, 4$, be pairwise non-isomorphic string modules. Then there exist irreducible morphisms $h_{i}: X_{i} \longrightarrow X_{i+1}$, with $i=$ $1,2,3$ such that $h_{3} h_{2} h_{1} \neq 0, h_{3} h_{2} h_{1} \in \Re^{4}\left(X_{1}, X_{4}\right), h_{2} h_{1} \notin \Re^{3}\left(X_{1}, X_{3}\right)$ and $h_{3} h_{2} \notin \Re^{3}\left(X_{2}, X_{4}\right)$ if and only if there exist an arrow $\beta_{0} \in\left(Q_{A}\right)_{1}$, a string $C=\gamma_{r}^{-1} \ldots \gamma_{1}^{-1} \beta_{0} \delta_{1}^{-1} \ldots \delta_{s}^{-1}$ that starts in a deep and ends on a 
peak (with $r, s \geq 0$ and $\gamma_{i}, \delta_{j}$ being arrows) with $\gamma_{1} \ldots \gamma_{r} \neq \delta_{s} \ldots \delta_{1}$ and satisfying one of the following conditions:

(a) $C$ does not start on a peak, $M(C)$ is non-injective and there exists a string $C^{\prime}$ that starts in a deep with $C^{\prime}=C \beta_{1}$ and $\beta_{1}=\gamma_{r}$ or $C^{\prime}=C \beta_{1} \nu_{1}^{-1} \ldots \nu_{t}^{-1}$ (with $\beta_{1}, \nu_{i}$ being arrows and $t \geq 0)$ such that either $e\left(\nu_{t}\right)=s\left(\gamma_{r}\right)$ or $\gamma_{r}^{-1} \ldots \gamma_{r-l}^{-1}=\nu_{t-l}^{-1} \ldots \nu_{t}^{-1}$ for some $l \in \mathbb{N}$, or if $t=0$ then $e\left(\beta_{1}\right)=s\left(\gamma_{r}\right)$.

(b) $C$ starts on a peak, $M(C)$ is non injective and $e\left(\delta_{s-1}\right)=s\left(\gamma_{r}\right)$ or $\gamma_{r}^{-1} \ldots \gamma_{r-l}^{-1}=\delta_{s-1-l}^{-1} \ldots \delta_{s-1}^{-1}$ for some $l \in \mathbb{N}_{0}$.

(c) $C$ does not end in a deep, $M(C)$ is non projective and there exists a string $C^{\prime}$ that ends on a peak with $C^{\prime}=\beta_{1} C$ and $\beta_{1}=\delta_{s} C$ or $C^{\prime}=\nu_{t}^{-1} \ldots \nu_{1}^{-1} \beta_{1} C$ (with $\beta_{1}, \nu_{i}$ being arrows and $t \geq 0)$ such that either $s\left(\delta_{s}\right)=e\left(\nu_{t}\right)$ or $\gamma_{r}^{-1} \ldots \gamma_{r-l}^{-1}=\nu_{s-l}^{-1} \ldots \nu_{t}^{-1}$ for some $l \in \mathbb{N}$ or if $t=0$ then $s\left(\delta_{s}\right)=e\left(\beta_{1}\right)$.

(d) $C$ ends in a deep, $M(C)$ is non projective and $e\left(\delta_{s}\right)=s\left(\gamma_{r-1}\right)$ or $\gamma_{r-1}^{-1} \ldots \gamma_{r-1-l}^{-1}=\delta_{s-l}^{-1} \ldots \delta_{s}^{-1}$ for some $l \in \mathbb{N}_{0}$.

Proof. Necessity. Observe that our assumption is equivalent to either (a) or (b) of (2.2). Assume we have condition (2.2)(a). Since $X_{2}$ is a string module and $\alpha\left(X_{1}\right)=1$ then, as recalled above, there exists an arrow $\beta_{0}$ such that $X_{2}=N\left(\beta_{0}\right)=M\left(\gamma_{r}^{-1} \ldots \gamma_{1}^{-1} \beta_{0} \delta_{1}^{-1} \ldots \delta_{s}^{-1}\right)$ where $C:=\gamma_{r}^{-1} \ldots \gamma_{1}^{-1} \beta_{0} \delta_{1}^{-1} \ldots \delta_{s}^{-1}$ is a string that starts in a deep and ends on a peak. Moreover, $X_{1}=U\left(\beta_{0}\right)=M\left(\gamma_{r}^{-1} \ldots \gamma_{1}^{-1}\right)$ and $\tau^{-1} X_{1}=$ $V\left(\beta_{0}\right)=M\left(\delta_{1}^{-1} \ldots \delta_{s}^{-1}\right)$. Note that $M(C)$ is not injective and that $\tau^{-1} X_{1} \not X_{1}$, since $\alpha\left(X_{1}\right)=1$ and $X_{4} \not X_{2}$. Then $\gamma_{1} \ldots \gamma_{r} \neq \delta_{s} \ldots \delta_{1}$.

Now, if $C$ does not start on a peak and since $C$ ends on a peak and $\alpha^{\prime}\left(X_{2}\right)=2$, by [4], the irreducible morphism $X_{2} \rightarrow X_{3}$ is the canonical embedding from $M(C)$ to $M\left(C^{\prime}\right)$, where $C^{\prime}$ a string starting in a deep of either one of these forms $C^{\prime}=C \beta_{1}$ or $C^{\prime}=C \beta_{1} \nu_{1}^{-1} \ldots \nu_{t}^{-1}$ (with $\beta_{1}, \nu_{i}$ being arrows and $t \geq 0$ ). Thus $X_{4}=M\left(C^{\prime \prime}\right)$, with $C^{\prime \prime}=\delta_{1}^{-1} \ldots \delta_{s}^{-1} \beta_{1}$ if $C^{\prime}=C \beta_{1}$ or $C^{\prime \prime}=\delta_{1}^{-1} \ldots \delta_{s}^{-1} \beta_{1} \nu_{1}^{-1} \ldots \nu_{t}^{-1}$ if $C^{\prime}=$ $C \beta_{1} \nu_{1}^{-1} \ldots \nu_{t}^{-1}$. By hypothesis, $\Re\left(X_{1}, X_{4}\right) \neq 0$ and $\beta_{1}=\gamma_{r}$, in the first case. Otherwise $e\left(\nu_{t}\right)=s\left(\gamma_{r}\right)$ or $\gamma_{r}^{-1} \ldots \gamma_{r-l}^{-1}=\nu_{t-l}^{-1} \ldots \nu_{t}^{-1}$ for some $l \in \mathbb{N}$, or if $t=0, e\left(\beta_{1}\right)=s\left(\gamma_{r}\right)$. Thus we get (a).

On the other hand, if $C$ starts on a peak, and since $C$ ends on a peak and $\alpha\left(X_{2}\right)=2$, by [4] the irreducible morphism $X_{2} \rightarrow X_{3}$ is the 
canonical projection $M(C) \rightarrow M\left(C^{\prime}\right)$ where $C^{\prime}=\gamma_{r}^{-1} \ldots \gamma_{1}^{-1} \beta_{0} \delta_{1}^{-1} \ldots \delta_{s-1}^{-1}$ is a string not starting in a deep. Moreover,

$$
0 \rightarrow M(C) \rightarrow \tau^{-1} X_{1} \oplus M\left(C^{\prime}\right) \rightarrow M\left(\delta_{1}^{-1} \ldots \delta_{s-1}^{-1}\right) \rightarrow 0
$$

is the almost split sequence starting at $X_{2}$. Since $\Re\left(X_{1}, X_{4}\right) \neq 0$, then $\gamma_{r}^{-1} \ldots \gamma_{r-l}^{-1}=\delta_{s-1-l}^{-1} \ldots \delta_{s-1}^{-1}$ for some $l \in \mathbb{N}$ or $e\left(\delta_{s-1}\right)=s\left(\gamma_{r}\right)$. Thus we get (b).

If we now assume that condition (2.2)(b) holds then dual arguments lead to the cases (c) and (d).

Sufficiency. By hypothesis, since there is an arrow $\beta_{0} \in\left(Q_{A}\right)_{1}$ such that $N\left(\beta_{0}\right)=M\left(\gamma_{r}^{-1} \ldots \gamma_{1}^{-1} \beta_{0} \delta_{1}^{-1} \ldots \delta_{s}^{-1}\right)$ where $C=\gamma_{r}^{-1} \ldots \gamma_{1}^{-1}$ $\beta_{0} \delta_{1}^{-1} \ldots \delta_{s}^{-1}$ starts in a deep and ends on a peak, then there is an almost split sequence in $\bmod A$ with indecomposable middle term of the form

$0 \rightarrow M\left(\gamma_{r}^{-1} \ldots \gamma_{1}^{-1}\right) \stackrel{f}{\rightarrow} M\left(\gamma_{r}^{-1} \ldots \gamma_{1}^{-1} \beta_{0} \delta_{1}^{-1} \ldots \delta_{s}^{-1}\right) \stackrel{g}{\rightarrow} M\left(\delta_{1}^{-1} \ldots \delta_{s}^{-1}\right) \rightarrow 0$.

Suppose that (a) holds. By [4](lemma p. 166), since there are arrows $\beta_{1}, \nu_{1}, \ldots, \nu_{t}$ such that $C^{\prime}=C \beta_{1}$ or $C^{\prime}=C \beta_{1} \nu_{1}^{-1} \nu_{t}^{-1}$ are strings starting in a deep, then the canonical embedding $M(C) \rightarrow M\left(C^{\prime}\right)$ is irreducible. In particular, $M\left(C^{\prime}\right) \not M\left(\delta_{1}^{-1} \ldots \delta_{s}^{-1}\right)$ and $M(C)$ is not injective. Then $\alpha^{\prime}(M(C))=2$ and there is a configuration of irreducible morphisms as in the condition (2.2)(a) as follows:

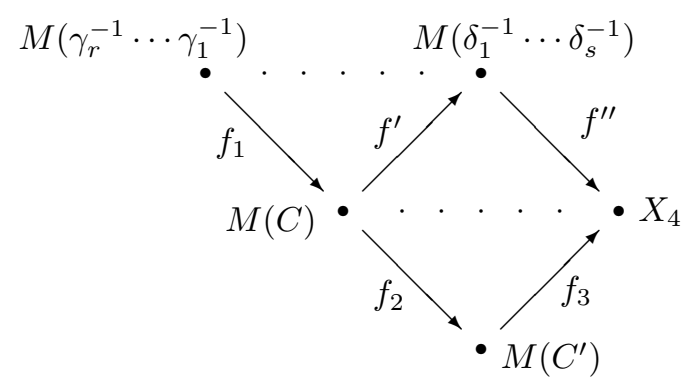

with $X_{4} \simeq M\left(\beta_{1} \delta_{1}^{-1} \ldots \delta_{s}^{-1}\right)$ or $X_{4} \simeq M\left(\nu_{t}^{-1} \ldots \nu_{1}^{-1} \beta_{1} \delta_{1}^{-1} \ldots \delta_{s}^{-1}\right)$ and $X_{1} \simeq M\left(\gamma_{r}^{-1} \ldots \gamma_{1}^{-1}\right)$. 
If $s\left(\nu_{t}\right)=e\left(\gamma_{r}\right)$ then $\operatorname{top}\left(M\left(\gamma_{r}^{-1} \ldots \gamma_{1}^{-1}\right)\right)=\operatorname{soc}\left(M\left(\nu_{t}^{-1} \ldots \nu_{1}^{-1} \beta_{1} \delta_{1}^{-1}\right.\right.$ $\left.\ldots \delta_{s}^{-1}\right)$ ). Therefore $\Re\left(X_{1}, X_{4}\right) \neq 0$.

Now, suppose that there is an $l \in \mathbb{N}$ such that $\gamma_{r}^{-1} \ldots \gamma_{r-l}^{-1}=\nu_{t-l}^{-1} \ldots \nu_{t}^{-1}$. Then there is an indecomposable module

$$
N=M\left(\gamma_{r}^{-1} \ldots \gamma_{r-l}^{-1}\right)=M\left(\nu_{t-l}^{-1} \ldots \nu_{t}^{-1}\right)
$$

and non-isomorphisms $\varphi_{1}=\pi f_{2} f_{1}$ where

$$
\pi: M\left(\gamma_{r}^{-1} \ldots \gamma_{1}^{-1} \beta_{0} \delta_{1}^{-1} \ldots \delta_{s}^{-1}\right) \rightarrow M\left(\gamma_{r}^{-1} \ldots \gamma_{r-l}^{-1}\right)
$$

is the canonical projection to $M\left(\gamma_{r}^{-1} \ldots \gamma_{r-l}^{-1}\right)$ and $\varphi_{2}=f_{3} \iota$ where

$$
\iota: M\left(\nu_{t-l}^{-1} \ldots \nu_{t}^{-1}\right) \rightarrow M\left(\gamma_{r}^{-1} \ldots \gamma_{1}^{-1} \beta_{1} \nu_{1}^{-1} \ldots \nu_{t}^{-1}\right)
$$

is the natural embedding. By [7](Theorem 2.4), since $N$ is not isomorphic to $M\left(C^{\prime}\right)$ and $\varphi_{1} \in \Re^{3}$, it is enough to show that $\varphi_{2} \varphi_{1} \neq 0$ to get the result. If however $\varphi_{2} \varphi_{1}=0$ then $f_{3} \iota \pi f_{2} f_{1}=0$ and hence

$$
\operatorname{Im}\left(\iota \pi f_{2} f_{1}\right) \subset \operatorname{Ker} f_{3} \subset \operatorname{Ker} f^{\prime \prime} \oplus \operatorname{Ker} f_{3}=\operatorname{Im}\left(f^{\prime}, f_{2}\right)^{t}
$$

and

$$
\operatorname{Im}\left(f^{\prime}, f_{2}\right)^{t}=M\left(\delta_{1}^{-1} \ldots \delta_{s}^{-1}\right) \oplus M\left(\gamma_{r}^{-1} \ldots \gamma_{1}^{-1} \beta_{0} \delta_{1}^{-1} \ldots \delta_{s}^{-1}\right)=X
$$

But $\operatorname{Im}\left(\iota \pi f_{2} f_{1}\right)=M\left(\gamma_{r}^{-1} \ldots \gamma_{r-l}^{-1}\right)$ is not a submodule of $X$ and then $\varphi_{2} \varphi_{1} \neq 0$, proving that $\Re\left(X_{1}, X_{4}\right) \neq 0$.

Now, suppose that condition (b) holds. Since, by hypothesis, $C$ starts on a peak and $C^{\prime}=\gamma_{r}^{-1} \ldots \gamma_{l}^{-1} \beta_{0} \delta_{1}^{-1} \ldots \delta_{s-1}^{-1}$ does not starts in a deep, then the canonical projection $M(C) \rightarrow M\left(C^{\prime}\right)$ is irreducible (see [4], lemma p. 169). Note that $M(C)$ is not injective, $\alpha^{\prime}(M(C))=2$ and the irreducible morphisms are as in (2.2)(a). By hypothesis, $e\left(\delta_{s}\right)=$ $s\left(\gamma_{r-1}\right)$ or there is an $l \in \mathbb{N}$ such that $\gamma_{r}^{-1} \ldots \gamma_{r-l}^{-1}=\delta_{s-l-1}^{-1} \ldots \delta_{s-1}^{-1}$. In the former case, $\operatorname{top} X_{1}=\operatorname{soc} X_{4}$. Therefore $\Re\left(X_{1}, X_{4}\right) \neq 0$. On the other hand, if there is an $l \in \mathbb{N}$ such that $\gamma_{r}^{-1} \ldots \gamma_{r-l}^{-1}=\delta_{s-l-1}^{-1} \ldots \delta_{s-1}^{-1}$, then there is an indecomposable module

$$
N=M\left(\gamma_{r}^{-1} \ldots \gamma_{r-l}^{-1}\right)=M\left(\delta_{s-l-1}^{-1} \ldots \delta_{s-1}^{-1}\right)
$$

and non-isomorphisms $\varphi_{1}=\pi f_{2} f_{1}$ where

$$
\pi: M\left(\gamma_{r}^{-1} \ldots \gamma_{1}^{-1} \beta_{0} \delta_{1}^{-1} \ldots \delta_{s-1}^{-1}\right) \rightarrow M\left(\gamma_{r}^{-1} \ldots \gamma_{r-l}^{-1}\right)
$$

is the canonical projection to $M\left(\gamma_{r}^{-1} \ldots \gamma_{r-l}^{-1}\right)$ and $\varphi_{2}=f_{3} \iota$ where

$$
\iota: M\left(\delta_{s-l-l}^{-1} \ldots \delta_{s-1}^{-1}\right) \rightarrow M\left(\gamma_{r}^{-1} \ldots \gamma_{1}^{-1} \beta_{0} \delta_{1}^{-1} \ldots \delta_{s-1}^{-1}\right)
$$


is the natural embedding. We claim that $0 \neq f_{2} f_{1}$ is a monomorphism, since $\operatorname{Im} f_{1} \bigcap \operatorname{Ker} f_{2}=\{0\}$. It is enough to show that $\varphi_{2} \varphi_{1} \neq 0$ to get the result. If however $\varphi_{2} \varphi_{1}=0$ then $f_{3} \iota \pi f_{2} f_{1}=0$ and hence

$$
\operatorname{Im}\left(\iota \pi f_{2} f_{1}\right) \subset \operatorname{Ker} f_{3} \subset \operatorname{Ker} f^{\prime \prime} \oplus \operatorname{Ker} f_{3}=\operatorname{Im}\left(f^{\prime}, f_{2}\right)^{t}
$$

and

$$
\begin{aligned}
\operatorname{Im}\left(f^{\prime}, f_{2}\right)^{t} & \simeq \tau^{-1} X_{1} \oplus X_{3} \\
& =M\left(\delta_{1}^{-1} \ldots \delta_{s}^{-1}\right) \oplus M\left(\gamma_{r}^{-1} \ldots \gamma_{1}^{-1} \beta_{0} \delta_{1}^{-1} \ldots \delta_{s-1}^{-1}\right) \\
& =X .
\end{aligned}
$$

But $\operatorname{Im}\left(\iota \pi f_{2} f_{1}\right)=M\left(\gamma_{r}^{-1} \ldots \gamma_{r-l}^{-1}\right)$ is not a submodule of $X$ and then $\varphi_{2} \varphi_{1} \neq 0$, proving that $\Re\left(X_{1}, X_{4}\right) \neq 0$.

If we assume that conditions (c) or (d) hold, with a similar analysis as before we get the condition (2.2)(b).

3.2. The above result allows us to characterize the quivers of the representation - finite string algebras having paths of irreducible morphisms between non-isomorphic indecomposable modules of length three with non-zero composite lying in the fourth power of the radical. We will illustrate it in the following example.

ExAmple. Let $A \simeq k Q_{A} / I_{A}$ be the $k$-algebra given by the quiver $Q_{A}$ :

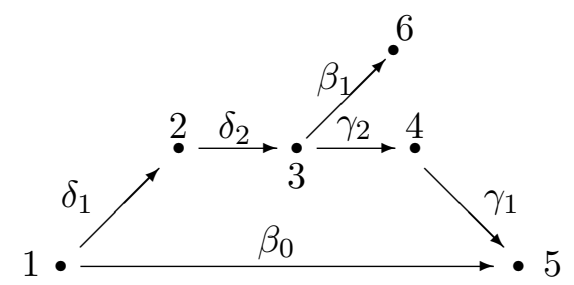

and $I_{A}$ generated by $\gamma_{2} \delta_{2}=0$ and $\beta_{1} \delta_{2}=0$.

The string $C=\gamma_{2}^{-1} \gamma_{1}^{-1} \beta_{0} \delta_{1}^{-1} \delta_{2}^{-1}$ starts in a deep and ends on a peak, since there is no arrow $\beta$ such that $C \beta^{-1}$ and $\beta^{-1} C$ are strings, respectively.

Observe that $C$ does not end in a deep because there is an arrow $\beta_{1}$ such that $\beta_{1} C=\beta_{1} \gamma_{2}^{-1} \gamma_{1}^{-1} \beta_{0} \delta_{1}^{-1} \delta_{2}^{-1}$ is a string. Moreover, $M(C)$ 
is non projective and $C^{\prime}=\beta_{1} C$ is a string that ends on a peak, with $\beta_{1} \neq \delta_{2}$. Then by (c), since $e\left(\delta_{2}\right)=s\left(\beta_{1}\right)$ we conclude that there is a path of three irreducible morphisms with non-zero composite in $\Re^{4}$.

Before stating our main result, we will prove the following lemma.

Lemma. Let $A \simeq k Q_{A} / I_{A}$ where $Q_{A}$ is the bypass:

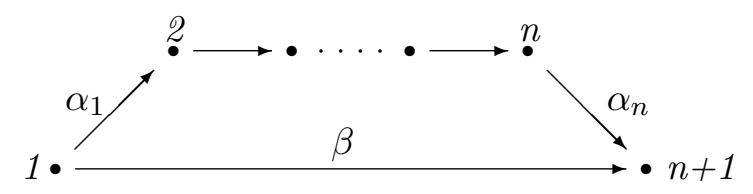

with $n \geq 2$, and $I_{A}$ is generated by a set of zero relations. Then, each pair of zero relations is overlapped in at least two arrows if and only if $J=\left\{i \in\left(Q_{A}\right)_{0}: \alpha_{n} \ldots \alpha_{i} \notin I_{A}\right.$ and $\left.\alpha_{i-1} \ldots \alpha_{1} \notin I_{A}\right\}$ is non-empty.

Proof. Assume that each pair of zero relations is overlapped in at least two arrows. Since $Q_{A}$ is a bypass, then all the relations have to share at least the same two arrows. We may assume that such two arrows are $\alpha_{j}$ and $\alpha_{j-1}$. Then clearly $\alpha_{n} \ldots \alpha_{j} \notin I_{A}$ and $\alpha_{j-1} \ldots \alpha_{1} \notin I_{A}$. Therefore, $j \in J$ and it is not empty.

Now, if $J \neq \emptyset$, then there are a minimum $j \in J$ and a maximum $m \in J$. Then $\alpha_{n} \ldots \alpha_{m} \ldots \alpha_{j} \alpha_{j-1} \in I_{A}$ and $\alpha_{m} \ldots \alpha_{j} \alpha_{j-1} \ldots \alpha_{1} \in$ $I_{A}$. We claim that $\alpha_{m} \ldots \alpha_{j-1}$ is a sub-path that belongs to each zero relation of $I_{A}$. In fact, assume that there is a zero relation $\gamma$ which does not have such a sub-path. Since $\gamma \in I_{A}$, then if $\alpha_{n}$ is an arrow that belongs to $\gamma$ we get a contradiction to the fact that $\alpha_{n} \ldots \alpha_{m} \ldots \alpha_{j} \notin I_{A}$. Otherwise, the relation $\alpha_{n} \ldots \gamma \in I_{A}$. Now if $\gamma=\alpha_{t} \ldots \alpha_{t-i}$ for $t-i \leq j$ we also get a contradiction with the fact that $\alpha_{n} \ldots \alpha_{m} \ldots \alpha_{j} \notin I_{A}$. In any other case we get a contradiction to the fact that $\alpha_{m-1} \ldots \alpha_{j} \alpha_{j-1} \ldots \alpha_{1} \notin I_{A}$.

We observe that if $m=j$ then there are exactly two arrows in common in each zero relation.

3.3. We now state and prove our main result.

TheOREM. Let $A=k Q_{A} / I_{A}$ be a connected triangular string algebra of finite representation type and $X_{i} \in$ indA pairwise non-isomorphic modules, for $i=1, \ldots, 4$. Then there exist irreducible morphisms 
$h_{i}: X_{i} \longrightarrow X_{i+1}$ such that $h_{3} h_{2} h_{1} \neq 0, h_{3} h_{2} h_{1} \in \Re^{4}\left(X_{1}, X_{4}\right), h_{2} h_{1} \notin$ $\Re^{3}\left(X_{1}, X_{3}\right)$ and $h_{3} h_{2} \notin \Re^{3}\left(X_{2}, X_{4}\right)$ if and only if one of the following conditions holds:

(a) The quiver $Q_{A}$ has a bypass $Q_{A}^{\prime}$ :

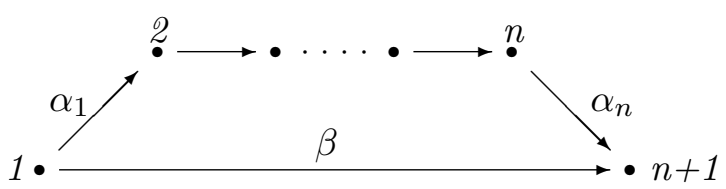

with $n \geq 2$, and such that $I_{A} \cap Q_{A}^{\prime}$ is generated by zero relations satisfying that the set $J=\left\{i \in\left(Q_{A}^{\prime}\right)_{0}: \alpha_{n} \ldots \alpha_{i} \notin\right.$ $I_{A}$ and $\left.\alpha_{i-1} \ldots \alpha_{1} \notin I_{A}\right\}$ is non-empty.

If $J=\{a\}$ then one of the conditions (i) or (ii) is satisfied.

(i) there exist an arrow $\delta \neq \alpha_{a}$ with $s(\delta)=a$. If there is an arrow $\delta^{\prime}$ such that $s\left(\delta^{\prime}\right)=e(\delta)$ then $\delta^{\prime} \delta \alpha_{a-1} \cdots \alpha_{1}=0$ and there are no arrow $\delta^{\prime \prime}$ with $e\left(\delta^{\prime \prime}\right)=e(\delta)$.

(ii) there exist an arrow $\mu \neq \alpha_{a-1}$ with $e(\mu)=a$. If there is an arrow $\delta^{\prime}$ such that $e\left(\delta^{\prime}\right)=s(\mu)$ then $\alpha_{n} \cdots \alpha_{a} \mu \delta^{\prime}=0$ and there are no arrow $\delta^{\prime \prime}$ with $s\left(\delta^{\prime \prime}\right)=s(\mu)$.

(b) The quiver $Q_{A}$ has a subquiver $Q_{A}^{\prime}$ :

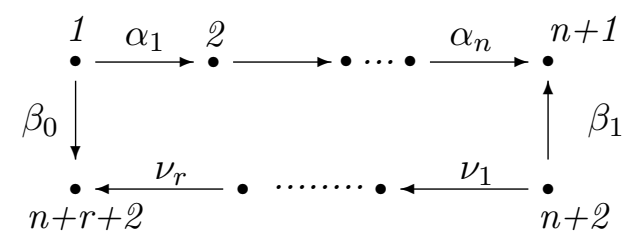

with $n \geq 2, r \geq 0$ and such that $Q_{A}^{\prime}$ is bounded by zero relations involving only arrows $\alpha_{i}$, satisfying that $J=\left\{i \in\left(Q_{A}^{\prime}\right)_{0}\right.$ : $\alpha_{n} \ldots \alpha_{i} \notin I_{A}$ and $\left.\alpha_{i-1} \ldots \alpha_{1} \notin I_{A}\right\}$ is non-empty. In case that $J=\{a\}$, we have that $\delta \alpha_{a-1} \cdots \alpha_{1}=0$ and $\alpha_{n} \cdots \alpha_{a} \delta=0$, for all arrows $\delta$ of $Q_{A}$.

(c) The quiver $Q_{A}$ has a subquiver $Q_{A}^{\prime}$ of the form:

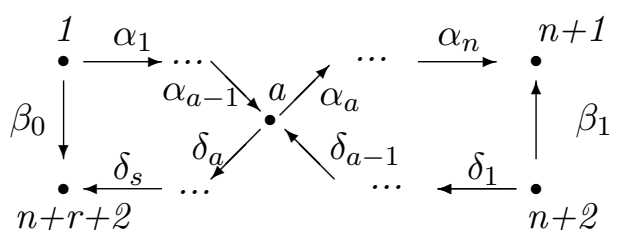


such that $I_{A} \cap Q_{A}^{\prime}$ is generated by zero relations, the set $J=$ $\left\{i \in\left(Q_{A}^{\prime}\right)_{0}: \alpha_{n} \ldots \alpha_{i} \notin I_{A}\right.$ and $\left.\alpha_{i-1} \ldots \alpha_{1} \notin I_{A}\right\}$ is non-empty, $\delta_{a} \alpha_{a-1}=0, \alpha_{a} \delta_{a-1}=0$ and $\delta_{s} \ldots \delta_{1} \notin I_{A}$. In case that $J=\{b\}$, with $a \neq b$, then $\delta \alpha_{b-1} \cdots \alpha_{1}=0$ and $\alpha_{n} \cdots \alpha_{b} \delta=0$, for all arrows $\delta$ of $Q_{A}$.

Proof. Necessity. By (3.1), there exists an arrow $\beta_{0} \in\left(Q_{A}\right)_{1}$ and a string $C=\gamma_{r}^{-1} \ldots \gamma_{1}^{-1} \beta_{0} \delta_{1}^{-1} \ldots \delta_{s}^{-1}$ that starts in a deep and ends on a peak with $\gamma_{1} \ldots \gamma_{r} \neq \delta_{s} \ldots \delta_{1}$.

Assume first that condition (a) of (3.1) holds. Then $C$ does not start on a peak, and there is an arrow $\beta_{1}$ such that $C \beta_{1}$ is a string. If $\beta_{1}=\gamma_{r}$ then $C^{\prime}=C \beta_{1}$ is a string that starts in a deep, and so we infer that there is a walk $Q_{A}^{\prime}$ in $Q_{A}$ as follows

$$
\stackrel{\beta_{1}}{\rightarrow} . \stackrel{\delta_{s}}{\leftarrow} \ldots \stackrel{\delta_{1}}{\leftarrow} \cdot \stackrel{\beta_{0}}{\rightarrow} \cdot \stackrel{\gamma_{1}}{\leftarrow} \ldots \stackrel{\gamma_{r}}{\leftarrow}
$$

Moreover, since $A$ is triangular and $C$ does not start on a peak, it implies that $Q_{A}^{\prime}$ is indeed a subquiver as follows

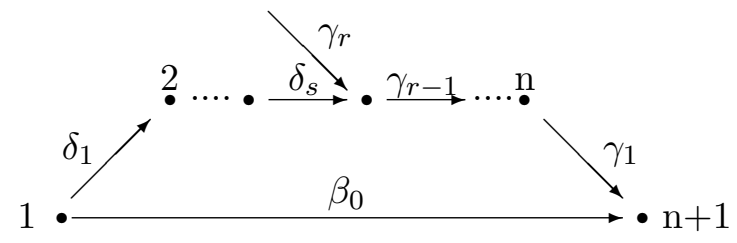

where $k Q_{A}^{\prime} \bigcap I_{A}=\langle\gamma>$ with $\ell(\gamma)=2$, because $A$ is a string algebra and $\gamma_{r-1} \gamma_{r} \neq 0$.

On the other hand, since $C$ ends on a peak, then $C^{\prime}$ ends on a peak and if there is an arrow $\delta$ such that $e(\delta)=s\left(\gamma_{r}\right)$ then $\gamma_{r} \delta \in I_{A}$. Observe that since $C^{\prime}=C \gamma_{r}$ is a string that starts in a deep then there is no arrow $\delta$ such that $s(\delta)=s\left(\gamma_{r}\right)$. Thus, (a) is satisfied.

Now, if $\beta_{1} \neq \gamma_{r}$ then $C^{\prime}$ is of the form

$$
C^{\prime}=\gamma_{r}^{-1} \ldots \gamma_{1}^{-1} \beta_{0} \delta_{1}^{-1} \ldots \delta_{s}^{-1} \beta_{1} \nu_{1}^{-1} \ldots \nu_{t}^{-1}
$$

and we infer that there is a walk $Q_{A}^{\prime}$ in $Q_{A}$ as follows

$$
\stackrel{\nu_{t}}{\leftarrow} \ldots \stackrel{\nu_{1}}{\leftarrow} . \stackrel{\beta_{1}}{\rightarrow} . \stackrel{\delta_{s}}{\leftarrow} \ldots \stackrel{\delta_{1}}{\leftarrow} . \stackrel{\beta_{0}}{\rightarrow} . \stackrel{\gamma_{1}}{\leftarrow} \ldots \stackrel{\gamma_{r}}{\leftarrow}
$$


with $t \geq 0$. If $t=0$ and $C^{\prime}$ is a string with the condition $e\left(\beta_{1}\right)=s\left(\gamma_{r}\right)$, then we infer that there is a walk $Q_{A}^{\prime}$ in $Q_{A}$ as follows

$$
\stackrel{\beta_{1}}{\rightarrow} . \stackrel{\delta_{s}}{\leftarrow} \ldots \stackrel{\delta_{1}}{\leftarrow} . \stackrel{\beta_{0}}{\rightarrow} . \stackrel{\gamma_{1}}{\leftarrow} \ldots \stackrel{\gamma_{r}}{\leftarrow} .
$$

Then it implies that $Q_{A}^{\prime}$ is a sub-quiver of the form:

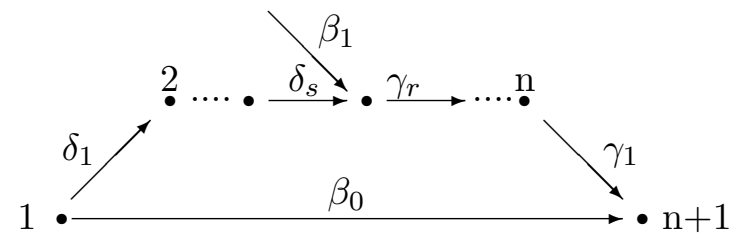

where $k Q_{A}^{\prime} \bigcap I_{A}=\langle\gamma>$ with $\ell(\gamma)=2$, since $C$ is a string that ends on a peak. Hence $J=\{a\}$ and $\gamma_{r} \delta_{s}=0$. Moreover, because $C$ ends on a peak then we also have that $\gamma_{r} \beta_{1}=0$. If there is an arrow $\delta^{\prime}$ such that $e\left(\delta^{\prime}\right)=s\left(\beta_{1}\right)$ then $\delta^{\prime} \beta_{1} \gamma_{r} \cdots \gamma_{1}=0$ since ${ }^{\prime} \beta_{1} \gamma_{r}=0$ and there are no arrow $\delta^{\prime \prime}$ with $s\left(\delta^{\prime \prime}\right)=s\left(\beta_{1}\right)$, since $C^{\prime}$ starts in a deep.

Now, if $t>0$ then by hypothesis, since $A$ is triangular, $C$ and $C^{\prime}$ are strings with the given conditions, $\gamma_{r}^{-1} \ldots \gamma_{r-l}^{-1}=\nu_{t-l}^{-1} \ldots \nu_{t}^{-1}$ or $e\left(\nu_{t}\right)=$ $s\left(\gamma_{r}\right)$, then it implies that $Q_{A}^{\prime}$ is indeed one of the following sub-quivers $(1),(2),(3),(4)$ or (5) below (identifying vertices in the cases that it is possible) and denoting the arrows $\nu_{1}, \ldots \nu_{t}, \gamma_{1}, \ldots, \gamma_{t}$ by $\alpha_{1}, \ldots, \alpha_{n}$ :

(1)

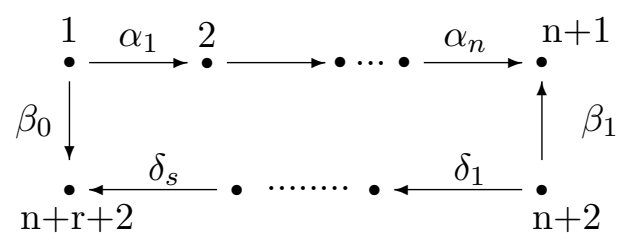

Observe that $\delta_{s} \ldots \delta_{1} \notin I_{A}$. In particular when $\delta_{s} \ldots \delta_{1}$ is a point then $\beta_{1} \beta_{0} \neq 0$ since $C^{\prime}$ is a string and (1) is the subquiver 
$(2)$

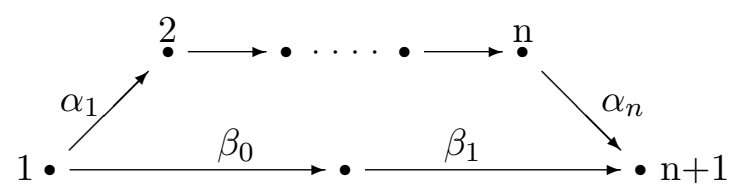

In both cases, since $C^{\prime}$ starts in a deep and ends on a peak then $k Q_{A}^{\prime} \cap I_{A}$ is non-zero and the set

$$
J=\left\{i \in\left(Q_{A}^{\prime}\right)_{0}: \alpha_{n} \ldots \alpha_{i} \notin I_{A} \text { and } \alpha_{i-1} \ldots \alpha_{1} \notin I_{A}\right\}
$$

is clearly non-empty. Observe that since $\delta_{s} \ldots \delta_{1} \notin I_{A}$ then $\alpha_{n} \ldots \alpha_{1}=0$. Suppose that $k Q_{A}^{\prime} \bigcap I_{A}=<\gamma>$ where $\ell(\gamma)=2$, then $J=\{a\}$. If $\delta \in\left(Q_{A}^{\prime}\right)_{1}$ with $s(\delta)=a$ and $\delta \neq \alpha_{a}$ then $\delta \alpha_{a-1} \cdots \alpha_{1} \in I_{A}$, because $C^{\prime}$ starts in a deep. Dually, if $\delta \in\left(Q_{A}^{\prime}\right)_{1}$ with $e(\delta)=a$ and $\delta \neq \alpha_{a-1}$ then $\alpha_{1} \cdots \alpha_{a} \delta \in I_{A}$ (observe that $C^{\prime}$ ends on a peak because $C$ ends on a peak). Therefore we get condition (b).

We can also identified some vertices in (1) getting the following subquiver in $Q_{A}$ :

(3)

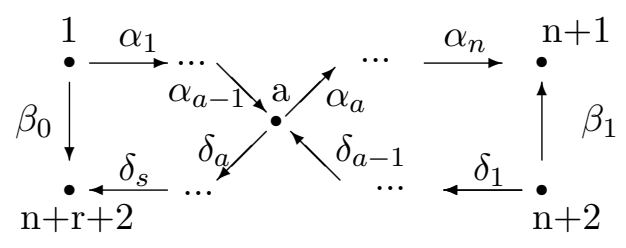

Since $C^{\prime}$ starts in a deep and ends on a peak and $\delta_{s} \ldots \delta_{1} \notin I_{A}$ then $k Q_{A}^{\prime} \cap I_{A}$ is non-zero and the set

$$
J=\left\{i \in\left(Q_{A}^{\prime}\right)_{0}: \alpha_{n} \ldots \alpha_{i} \notin I_{A} \text { and } \alpha_{i-1} \ldots \alpha_{1} \notin I_{A}\right\}
$$

is non-empty. Moreover, since $A$ is a representation-finite string algebra, then $\delta_{a} \alpha_{a-1}=0$ and $\alpha_{a} \delta_{a-1}=0$. Doing a similar analysis as the one made for the subquivers (1) and (2) when $J=\{a\}$ we get (c).

Besides the quivers above, we have the following possibilities 
$(4)$

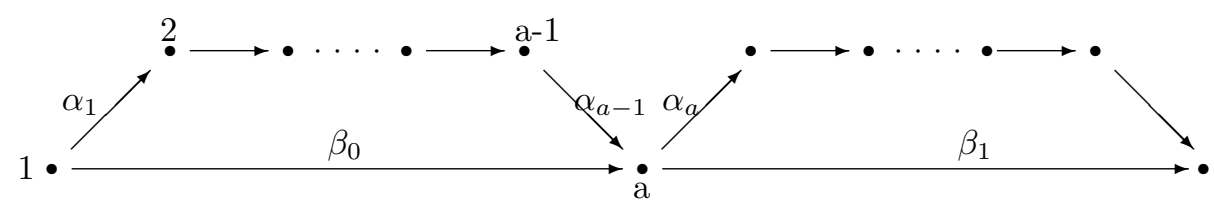

and

(5)

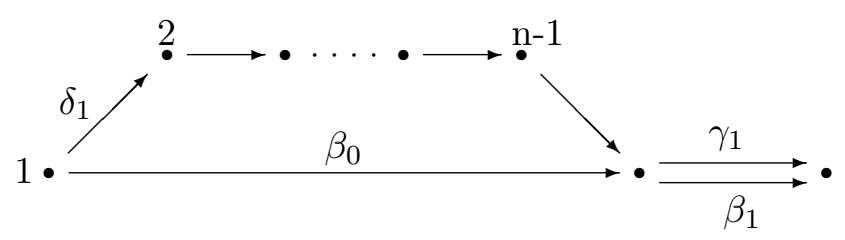

which gives, clearly, representation-infinite algebras, a contradiction to our hypothesis.

Now, assume that condition (b) of (3.1) holds. Then

$$
C=\gamma_{r}^{-1} \ldots \gamma_{1}^{-1} \beta_{0} \delta_{1}^{-1} \ldots \delta_{s}^{-1}
$$

is a string that starts on a peak, and we infer that there is a walk $Q_{A}^{\prime}$ in $Q_{A}$ of the form:

$$
\stackrel{\delta_{s}}{\leftarrow} \ldots \stackrel{\delta_{1}}{\leftarrow} \cdot \stackrel{\beta_{0}}{\rightarrow} \cdot \stackrel{\gamma_{1}}{\leftarrow} \ldots \stackrel{\gamma_{r}}{\leftarrow}
$$

First, if $e\left(\delta_{s-1}\right)=s\left(\gamma_{r}\right)$ and since $A$ is triangular then we get a subquiver as follows

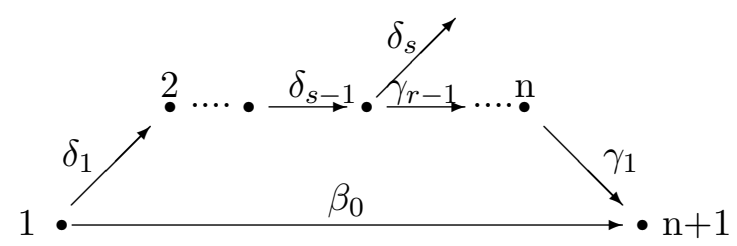


where $\delta_{s} \ldots \delta_{1} \notin I_{A}$ and $k Q_{A}^{\prime} \bigcap I_{A}=<\gamma>$ with $\ell(\gamma)=2$, since $C$ ends on a peak and $A$ is a string algebra. Moreover, by the conditions of the strings $C$ and $C^{\prime}$, we have that the conditions of (a)(i) hold.

If $\gamma_{r}^{-1} \ldots \gamma_{r-l}^{-1}=\delta_{s-1-l}^{-1} \ldots \delta_{s-1}^{-1}$ for some $l \geq 0$ then $\gamma_{r-l-1}=\delta_{s}$, since otherwise we have a subquiver of the form:

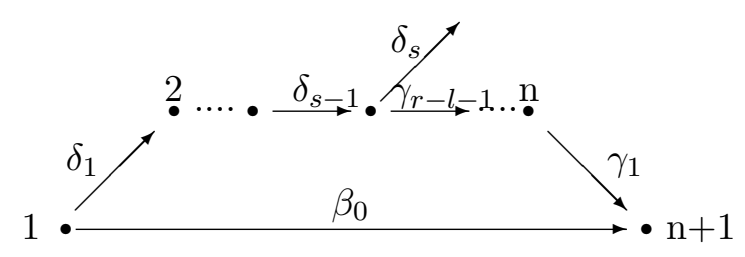

with $\delta_{s} \delta_{s-1} \in I_{A}$ since $A$ is a string algebra, a contradiction to the fact that $C$ is a string. Moreover, we proved that if $\gamma_{r}^{-1} \ldots \gamma_{r-l}^{-1}=$ $\delta_{s-1-l}^{-1} \ldots \delta_{s-1}^{-1}$ then $\gamma_{r}^{-1} \ldots \gamma_{r-l}^{-1} \gamma_{r-l-1}^{-1}=\delta_{s-1-l}^{-1} \ldots \delta_{s-1}^{-1} \delta_{s}^{-1}$, for some $l \geq 0$. Then $Q_{A}^{\prime}$ is indeed a bypass as follows

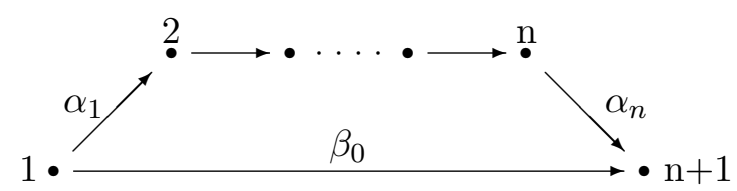

with $\ell(\gamma)>2$. Denote by $a=\min J$ and $b=\max J$. Since $C$ starts on a peak, there are no arrow ending in $b$, but anyway we can also have three irreducible morphisms with non zero composite in $\Re^{4}$ when we consider the string given in $(3.1)(d)$. In this case, we find that such irreducible morphisms are in a configuration as described in (2.2)(b), proving the implication.

Now, if (3.1)(c) holds, since $C$ does not ends on a peak, then there is an arrow $\beta_{1}$ such that $\beta_{1}^{-1} C$ is a string. In a similar way as before we can prove that conditions (c) or (d) of (3.1) imply (2.2)(a) and $(2.2)(\mathrm{b})$, which finishes this implication.

Sufficiency. To prove this implication, we are going to show that for each subquiver stated, we can find strings as in Proposition 3.1. Since we get the subquivers using the fact that there has to be a particular string on it, then clearly we can recover it from the quiver proving 
all the cases. We are going to prove here only one case to show the technique and we left the other cases to the reader.

Assume that (a) holds. First, consider the case that $b=\max J$, $a=\min J$ and $a \neq b$. Then there is an $l>0$ such that $\gamma_{r-l} \ldots \gamma_{r}=$ $\delta_{s-1} \ldots \delta_{s-1-l}$ and we have a string:

$$
C: b \stackrel{\delta_{s}}{\leftarrow} \ldots \stackrel{\delta_{1}}{\leftarrow} . \stackrel{\beta_{0}}{\rightarrow} . \stackrel{\gamma_{1}}{\leftarrow} \ldots \stackrel{\gamma_{r}}{\leftarrow} a
$$

that starts in a deep and ends on a peak. Moreover, $C$ is a string starting on a peak, and (3.1)(b) holds. Now, if $\max J=\min J=a$, then the string

$$
C: b \stackrel{\delta_{s}}{\leftarrow} \ldots \stackrel{\delta_{1}}{\leftarrow} . \stackrel{\beta_{0}}{\rightarrow} . \stackrel{\gamma_{1}}{\leftarrow} \ldots \stackrel{\gamma_{r}}{\leftarrow} a
$$

starts in a deep and ends on a peak. Moreover, by hypothesis if (i) holds then $C$ is a string not starting on a peak. Then there is an arrow $\beta_{1}$ and a string $C^{\prime}=C \beta_{1}$ or $C^{\prime}=C \beta_{1} \nu_{1}^{-1} \ldots \nu_{t}^{-1}$ that starts in a deep. Clearly, $M(C)$ is not injective and we get condition (3.1)(a).

In a similar way, we can analyzed the rest of the cases getting the result.

3.4. In [6], we gave a characterization of the quivers of triangular string algebras of finite representation type having two irreducible morphisms between indecomposable modules with non zero composite in $\Re^{3}$. Using this result and the above theorem we can obtain a characterization of the quivers of triangular string algebras of finite representation type having three irreducible morphisms between nonisomorphic indecomposable modules with non zero composite in $\Re^{4}$, but not two with non-zero composite in $\Re^{3}$.

Corollary. Let $A=k Q_{A} / I_{A}$ be a connected triangular string algebra of finite representation type and $X_{i} \in$ indA pairwise nonisomorphic modules, for $i=1, \ldots, 4$. Then there are irreducible morphisms $h_{i}: X_{i} \rightarrow X_{i+1}$ for $i=1,2,3$ with non-zero composite in $\Re^{4}\left(X_{1}, X_{4}\right)$ and for any two irreducible morphisms we have that their composite is not in $\Re^{3}$ if and only if the quiver $Q_{A}$ does not have a bypass as in (3.2)(d) and one of the following holds: 
(a) The quiver $Q_{A}$ has a bypass $Q_{A}^{\prime}$ :

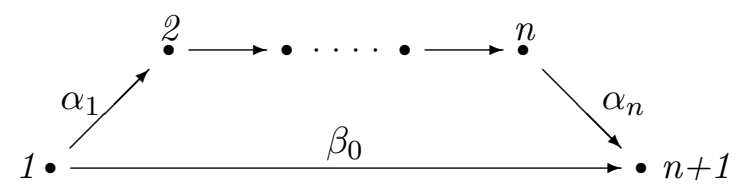

where $I_{A} \cap Q_{A}^{\prime}$ is generated by zero relations such that the set $J=\left\{i \in\left(Q_{A}^{\prime}\right)_{0}: \alpha_{n} \ldots \alpha_{i} \notin I_{A}\right.$ and $\left.\alpha_{i-1} \ldots \alpha_{1} \notin I_{A}\right\}=\{a\}$ and satisfying one of the following conditions:

(i) there exist an arrow $\delta \neq \alpha_{a}$ with $s(\delta)=a$. If there is an arrow $\delta^{\prime}$ such that $s\left(\delta^{\prime}\right)=e(\delta)$ then $\delta^{\prime} \delta=0$ and there are no arrow $\delta^{\prime \prime}$ with $e\left(\delta^{\prime \prime}\right)=e(\delta)$.

(ii) there exist an arrow $\mu \neq \alpha_{a-1}$ with $e(\mu)=a$. If there is an arrow $\delta^{\prime}$ such that $e\left(\delta^{\prime}\right)=s(\mu)$ then $\mu \delta^{\prime}=0$ and there are no arrow $\delta^{\prime \prime}$ with $s\left(\delta^{\prime \prime}\right)=s(\mu)$.

(b) The quiver $Q_{A}$ has a sub-quiver $Q_{A}^{\prime}$ :

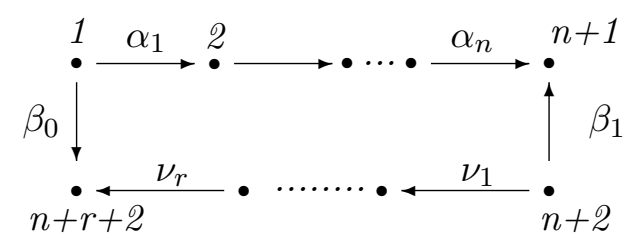

with $n \geq 2$ and $r \geq 0$ bounded by zero relations involving only arrows $\alpha_{i}$ satisfying that the set $J=\left\{i \in\left(Q_{A}^{\prime}\right)_{0}: \alpha_{n} \ldots \alpha_{i} \notin\right.$ $I_{A}$ and $\left.\alpha_{i-1} \ldots \alpha_{1} \notin I_{A}\right\}$ is non-empty. In case that $J=\{a\}$, then $\delta \alpha_{a-1} \cdots \alpha_{1}=0$ and $\alpha_{1} \cdots \alpha_{a} \delta=0$, for all arrows $\delta$ in $Q_{A}$.

(c) The quiver $Q_{A}$ has a subquiver $Q_{A}^{\prime}$ :

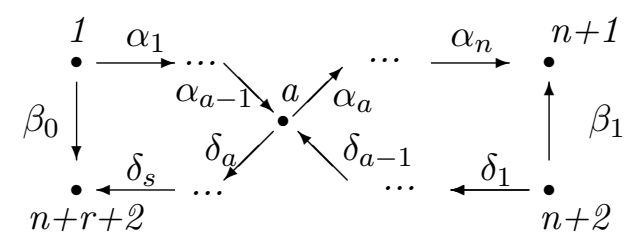

with $Q_{A}^{\prime}$ bounded by zero relations, satisfying that $J=\{i \in$ $\left(Q_{A}^{\prime}\right)_{0}: \alpha_{n} \ldots \alpha_{i} \notin I_{A}$ and $\left.\alpha_{i-1} \ldots \alpha_{1} \notin I_{A}\right\}$ is non-empty, 
$\delta_{a} \alpha_{a-1}=0, \alpha_{a} \delta_{a-1}=0$ and $\delta_{s} \ldots \delta_{1} \notin I_{A}$. In case that $J=\{b\}$, with $b \neq a$, then $\delta \alpha_{b-1} \cdots \alpha_{1}=0$ and $\alpha_{n} \cdots \alpha_{b} \delta=0$, for all arrows $\delta$ of $Q_{A}$.

Proof. The result follows easily from (3.3) and [6](Proposition 3.4).

3.5. We finish this paper with some examples.

ExAmples. (a) Let $A$ be the algebra given by the quiver:

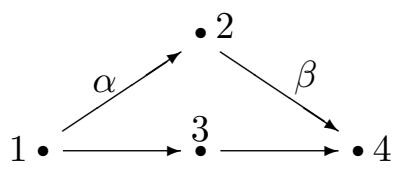

with $\beta \alpha=0$

For this algebra, there are paths of three irreducible morphisms whose composition is non-zero but lying in the fourth power of the radical. On the other hand, there are no pairs of irreducible morphisms whose composite is non-zero and lies in $\Re^{3}$. We refer to [8] for details on the latter remark.

(b) Let now $A$ be the $k$-algebra given by the quiver:

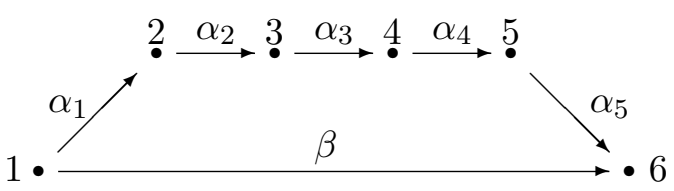

with $\alpha_{5} \alpha_{4} \alpha_{3} \alpha_{2}=0$ and $\alpha_{4} \alpha_{3} \alpha_{2} \alpha_{1}=0$. Denoting by $P_{j}$ (or $I_{j}$ ) the indecomposable projective (injective, respectively) associated with the vertex $j$, then there are almost split sequences

$$
\begin{gathered}
0 \longrightarrow P_{3} \stackrel{f_{1}}{\longrightarrow} M_{1} \stackrel{h_{1}}{\longrightarrow} I_{4} \longrightarrow 0 \text { and } \\
0 \longrightarrow M_{1} \stackrel{\left(h_{1} ; f_{2}\right)^{t}}{\longrightarrow} I_{4} \oplus M_{2} \stackrel{\left(h_{2}, f_{3}\right)}{\longrightarrow} I_{3} \longrightarrow 0
\end{gathered}
$$

where $M_{1}, M_{2} \in \operatorname{ind} A$. Observe that $\Re\left(P_{3}, I_{4}\right) \neq 0$, and so, by [6] (2.4) there are irreducible morphisms $P_{3} \stackrel{g_{1}}{\longrightarrow} M_{1} \stackrel{g_{2}}{\longrightarrow} I_{4}$ with a non-zero composite in $\Re^{3}$. Also, since $\Re\left(M_{2}, M_{2}\right)$ has a non-zero morphism $\varphi$, 
one can easily see (as in the above example) that the composite of the irreducible morphisms $f_{3}, f_{2}+\varphi f_{2}$ and $f_{1}$ is non-zero and lies in $\Re^{4}$.

\section{References}

1. E. Alvares, F. U. Coelho, A note on the composite of two irreducible morphisms, Comm. Algebra 37,6 (2009) 2097-2099.

2. I. Assem, D. Simson, A. Skowroński, Elements of representation theory of associative algebras. London Math. Soc. Student texts 65. Cambridge University Press, 2006.

3. M. Auslander, I. Reiten, S. Smalø, Representation Theory of Artin Algebras, vol 36 of Cambridge Studies in Advanced Mathematics, Cambridge University Press, 1995.

4. M. Butler, C. Ringel, Auslander-Reiten sequences with few middle terms and applications to string algebras, Comm. Algebra, 15 (1987), 145-179.

5. C. Chaio, Sobre la composición de morfismos irreducibles y las potencias del radical, PhD. Thesis, Universidad Nacional del Sur. Bahía Blanca (2006).

6. C. Chaio, F. U. Coelho, S. Trepode, On the composite of two irreducible morphisms in radical cube, J. Algebra 312 (2007), 650-667.

7. C. Chaio, F. U. Coelho, S. Trepode, On the composite of irreducible morphisms in almost-sectional paths, J. Pure Appl. Algebra 212 (2008), 244264.

8. C. Chaio, F. U. Coelho, S. Trepode, On the composite of three irreducible morphisms in the fourth power of the radical, Communications in Algebra, to appear.

9. K. Igusa, G. Todorov, A characterization of finite Auslander-Reiten quivers, J. Algebra 89 (1984) 148-177.

10. S. Liu, Degrees of irreducible maps and the shapes of Auslander-Reiten quivers, J. London Math. Soc (2) 45 (1992) 32-54.

11. C. M. Ringel, Tame algebras and integral quadratic forms, Lecture Notes in Math., vol 1099, Springer-Verlag, Berlin-Heidelberg, 1984. 